\title{
Increase of non- HBV, non- HCV related hepatocellular carcinoma -Over 20 years single institution study-
}

\section{Yuko Nagaoki}

Hiroshima University

Hideyuki Hyogo

Hiroshima University

Yuwa Ando

Hiroshima University

Yumi Kosaka

Hiroshima University

Shinsuke Uchikawa

Hiroshima University

\section{Yuno Nishida}

Hiroshima University

\section{Yuji Teraoka}

Hiroshima University

\section{Kei Morio}

Hiroshima University

Hatsue Fujino

Hiroshima University

Atsushi Ono

Hiroshima University

\section{Takashi Nakahara}

Hiroshima University

\section{Eisuke Murakami}

Hiroshima University

\section{Masami Yamauchi}

Hiroshima University

\section{Wataru Okamoto}

Hiroshima Universty

\section{Tomokazu Kawaoka}

Hiroshima University

\section{Masataka Tsuge}


Hiroshima University

\section{Akira Hiramatsu}

Hiroshima University

\section{Daiki Miki}

Hiroshima University

\section{Michio Imamura}

Hiroshima University

\section{Shoichi Takahashi}

Hiroshima University

\section{Kazuaki Chayama}

Hiroshima University

Hiroshi Aikata ( $\square$ aikata@hiroshima-u.ac.jp )

\section{Research article}

Keywords: HCC, NBNC-HCC, NASH, cryptogenic

Posted Date: September 21st, 2020

DOI: https://doi.org/10.21203/rs.3.rs-51308/v2

License: (c) (1) This work is licensed under a Creative Commons Attribution 4.0 International License.

Read Full License

Version of Record: A version of this preprint was published at BMC Gastroenterology on July 31st, 2021. See the published version at https://doi.org/10.1186/s12876-021-01884-5. 


\section{Abstract}

Background: We previously reported the trends of etiologies of hepatocellular carcinoma (HCC) between 1995 and 2009. By updating this study, we aimed to evaluate the incidence of HCC with non- hepatitis B viral and non- hepatitis C viral (NBNC) etiologies and explore the clinical characteristics, also.

Methods: Consecutive 2171 HCC patients at our institution were enrolled between 1992 and 2018 in this retrospective cohort study. A comparative study was conducted by dividing into two groups, an early group from 1992 to 2009 and a late group from 2010 to 2018.

Results: NBNC-HCC account for 514 patients (23.6\%). The proportion of NBNC-HCC has continued to increase from $26.5 \%$ in 2009 to $46.3 \%$ in 2018. NBNC-HCC were getting older (median ages, 67 to 73 years). Type 2 diabetes mellitus ( $48.5 \%$ to $60.3 \%$ : $P=0.008$ ), hypertension ( $48.5 \%$ to $57.4 \%$ : $P=0.047$ ), and hyperlipidemia (39.2\% to $53.8 \%$ : $P=0.001$ ) has increased significantly in recent years. The median FIB-4 index have decreased (4.37 to 3.61: $P=0.026$ ) and the median platelet counts have increased (15.1 to 17.9: $P=0.013$ ) significantly. Among 514 NBNC-HCC, 194 patients underwent hepatic resection in which $14.9 \%, 29.3 \%$, and $55.8 \%$ were based on nonalcoholic steatohepatitis (NASH), alcoholic liver disease (ALD), and cryptogenic, respectively. Cirrhosis was detected in $72.4 \%, 38.6 \%$, and $15.5 \%$ of NASH, ALD and cryptogenic, respectively. The prevalence of cirrhosis in NASH were significantly higher than other groups $(P<0.001)$, overall, $70 \%$ of the background liver of NBNC-HCC was not cirrhosis. On the other hands, the median FIB-4 index in cryptogenic HCC were 2.56 and significantly lower than other groups, FIB-4 index considered as one of useful screening of HCC.

Conclusions: NBNC-HCC has rapidly been increasing in recent years even in regional university hospital. The presence of metabolic syndrome may be important risk factors. Furthermore, HCC from non-cirrhotic liver needs attention carefully. Accordingly, it is speculated that these patients should receive periodic surveillance for HCC development.

\section{Background}

Hepatocellular carcinoma (HCC) is one of the most common malignant tumors in the world. In Japan, it ranks fifth in men and sixth in women as a cause of death from malignancies [1] and $93.3 \%$ of primary liver cancers are HCC [2]. Until now, hepatitis $C$ virus (HCV) and hepatitis B virus (HBV) has contributed to primary liver cancer, especially the development of HCC. That is, HCV-related HCC account for $67 \%$ of all HCCs followed by HBV- related HCC that account for $16 \%$ of all HCC in the vast majority of such cases worldwide [3].

However, because of efforts to control HBV and HCV infections, mortality rates have started to decrease in Asia and Southern Europe [4]. In those with active chronic HBV infection, long-term suppression using nucleotide analogs may reduce the incidence of HBV-related HCC $[5,6]$, and the eradication of HCV by antiviral therapy can reduce HCV-related HCC [7]. 
According to our previous report [8], $17 \%$ and $67 \%$ of HCC were related to HBV-HCC and HCV-HCC, respectively. Fifteen percent of that was negative for hepatitis $B$ surface antigen (HBsAg) and antibody to $\mathrm{HCV}$ (HCVAb) (NBNC-HCC). Especially, the incidence of HCV-related HCC has gradually decreased in recent years, whereas the incidence of HCCs associated with NBNC- HCC has gradually increased from 10 $\%$ in 1995 to $19 \%$ in 2009 [8]. Furthermore, Tateishi et al. reported that with NBNC-HCC has increased from $10 \%$ in 1991 to $32.5 \%$ in $2015[9,10]$.

In addition to alcoholic liver disease (ALD), which was reported as risk factors previously, it is recently becoming known that nonalcoholic fatty liver disease (NAFLD).

In ALD, $20 \%$ to $40 \%$ of alcoholics develop fibrosis, $10-20 \%$ eventually progress to cirrhosis [11], approximately $3 \%$ of patients progress to cirrhosis annually [12], 1-2\% of cirrhosis are diagnosed with HCC every year [13].

Recently, impact of NAFLD or non-alcoholic fatty steatohepatitis (NASH) as the hepatic manifestation of diabetes and obesity has been clearly linked to progression of liver fibrosis, cirrhosis and HCC risk in several studies [14-16]. Recent evidence indicates that obesity and T2DM are HCC risk factors [17-20]. In Japan, overweight and obese indicates a relative risk of 1.74 compared with normal and low-weight individuals [21]. Moreover, many studies have reported that T2DM is the risk of HCC, revealed a 2-4-fold greater risk of HCC among patients with T2DM compared with nondiabetic controls [22-25]. Insulin resistance (IR) associated with metabolic syndrome, T2DM and NAFLD leads to increased release of free fatty acids from adipocytes and the release of multiple pro-inflammatory cytokines including tumor necrosis factor alpha (TNF-a), interleukin-6 (IL-6), leptin, and resistin, as well as decreased amounts of adiponectin, and obesity is characterized by a low-grade chronic inflammatory response that is associated with increased in HCC [14, 26-29]. T2DM, obesity, and the metabolic syndrome have been growing in prevalence and are independent risk factors for the development of HCC. In this way, many studies have been reported on the risk of NBNC HCC.

These characteristics were similar to our previous report [8], the transition and risk factors in NBNCHCC were examined.

On the other hands, whereas various causes influence the development of NBNC-HCC, few reports have examining characteristics such as background liver using pathological specimens obtained by hepatectomy. Several studies have reported that NASH can progress to cirrhosis, advanced fibrosis are important risk factors for HCC [16, 30-32], and the prevalence of cirrhosis in the ALD-HCC was significantly higher, too $[11,17,33-36]$. NASH and ALD have now become the leading indications for liver transplantation such as HCV and HBV.

Recently, the increase in the number of NBNC-HCC related to NAFLD, ALD, obesity, diabetes, and metabolic syndrome are becoming a serious problem also in Japan. However, the clinical features of such HCC including those of NBNC-HCC are not fully elucidated in Japan. Therefore, we updated the data 
and investigated the clinical characteristics of NBNC-HCC by employing liver specimens pathologically because of urgent needs for the prevention and the future strategies against NBNC-HCC.

\section{Methods}

\section{Patients}

We retrospectively reviewed 2171 consecutive patients of primary HCC diagnosed at Hiroshima University Hospital from January 1992 to December 2018. All subjects gave informed consent to participate in the study according to the process approved by the ethical committee in Hiroshima University Hospital and conforming to the ethical guidelines of the Declaration of Helsinki.

\section{Laboratory Evaluation}

We collected the detailed clinical data (age, gender, history of habitual alcohol consumption, height, weight, lifestyle-related diseases, and laboratory parameters).

Biochemical data were measured using standard techniques for each patient at the time of diagnosis of primary HCC. Fibrosis (FIB)-4 index were calculated as a surrogate marker of liver fibrosis [37]. FIB-4 index $=$ age $($ years $) \times$ aspartate aminotransferase $($ AST $)[I U / L] /\left(\right.$ platelet count $\left[10^{9} / \mathrm{L}\right] \times($ ALT $\left.[I U / L]){ }^{1 / 2}\right)$. The albumin-bilirubin (ALBI) grade were used as assessment tools for hepatic reserve function. ALBI grade was calculated based on serum albumin and total-bilirubin values using the following formula: ALBIscore: $(\log 10$ bilirubin $(\mu \mathrm{mol} / \mathrm{L}) \times 0.66)+($ albumin $(\mathrm{g} / \mathrm{L}) \times-0.085)$; and ALBI grade was defined by the score of the following: $\leq-2.60=$ Grade $1,>-2.60$ to $\leq-1.39=$ Grade $2,>-1.39=$ Grade 3 [38].

HBV-HCC was defined as positive for HBsAg and negative for HCVAb, HCV-HCC was defined as positive for HCVAb and negative for HBsAg, NBNC-HCC was defined as negative for both HBsAg and HCVAb.

Alcohol liver disease (ALD) was determined when alcohol intake was more than $60 \mathrm{~g} /$ day for five years [39]. The body mass index (BMI) was calculated as body weight in kilograms divided by the square of the height in meters $\left(\mathrm{kg} / \mathrm{m}^{2}\right)$. The $\mathrm{HbA} 1 \mathrm{c}(\%)$ value was estimated as a National Glycohemoglobin Standardization Program (NGSP) equivalent value (\%) calculated by using the formula: A1c (\%) = A1c (Japan Diabetes Society (JDS)) (\%) $+0.4 \%$, considering the relational expression of A1c (JDS) (\%) measured by the previous Japanese standard substance and measurement methods and HbA1c (NGSP) [40]. Type 2 diabetes mellitus (T2DM) was diagnosed in cases with a high fasting plasma glucose (FPG, $\geq 126 \mathrm{mg} / \mathrm{dL}$ ), high HbA1c (NGSP) (HbA1c, $\geq 6.5 \%$ ), use of glucose-lowering agents, or a self-reported history of a clinical diagnosis.

Hyperlipidemia was diagnosed when the patient was being treated with lipid-lowering medications or had elevated levels of total cholesterol higher than $220 \mathrm{mg} / \mathrm{dL}$ and/or the triglyceride level was over 150 $\mathrm{mg} / \mathrm{dL}$. Hypertension was diagnosed when the patient was on antihypertensive medications and/or had a resting recumbent blood pressure of $130 / 85 \mathrm{mmHg}$ or more on at least two occasions. 


\section{Pathological evaluation}

The pathological status of underlying liver disease was based on microscopic examination of the noncancerous part of the surgical specimen or biopsy specimen with hematoxylin-eosin and Azan staining. All liver tissue specimens were evaluated by two senior pathologists who were unaware of the laboratory data and the clinical course. Histological diagnosis of NASH was confirmed according to Brunt's criterion $[41,42]$. Steatosis was graded as follows: grade 1 ( $\geq 5 \%$ and $<33 \%$ of hepatocytes affected); grade 2 (33$66 \%$ of hepatocytes affected); or grade 3 ( $66 \%<$ of hepatocytes affected). Necroinflammation was graded 0 (absent) to 3 (1, occasional ballooned hepatocytes and no or very mild inflammation; 2 , ballooning of hepatocytes and mild-to-moderate portal inflammation; 3 , intra-acinar inflammation and portal inflammation). Fibrosis was graded 0 (absent) to 4 (1, perisinusoidal/pericellular fibrosis; 2 , periportal fibrosis; 3 , bridging fibrosis; 4 , cirrhosis). Ballooning was graded 0 (none) to 2 ( 1 , few balloon cells; 2 , many cells/prominent ballooning) [41, 42].

The diagnosis of HCC was based on the hypervascular staining pattern of the arterial phase and the hypovascular staining pattern of the portal phase, and was confirmed by dynamic CT, magnetic resonance imaging, and/or angiography [43]. Tumors without enhancement upon imaging were diagnosed by fine-needle biopsy. Clinical staging (TNM classification) was performed according to the criteria of the Liver Cancer Study Group of Japan [44].

\section{Statistical analysis}

Results are shown as median values or percentages. Continuous variables are reported as median deviation and were compared using the Mann-Whitney U-test. Categorical variables were compared using the chi-square or Fisher exact test, as appropriate. The Cochran Armitage trend test was used to evaluate increasing or decreasing trends. All statistical analyses were performed using the SPSS software package (version SPSS 23.0, IBM, Armonk, NY, USA). A P value $<0.05$ was considered a statistically significant difference.

\section{Results}

\section{Trend of etiology of liver diseases in patients with HCC}

Among 2171 patients with primary HCC from 1992 to 2018, 361 patients (16.6\%) and 1271 patients (58.5\%) were related to HBV-HCC and HCV-HCC, respectively (Fig.1). Five hundred and fourteen patients (23.6\%) of that was negative for HBsAg and HCVAb (NBNC-HCC). Twenty-five (1.1\%) patients with HCC were positive for both HBsAg and HCVAb (BC-HCC). In order to compare with the previous reports up to 2009 , the study was divided into three periods.

The etiologies of liver diseases in patients with HCC in three periods were as follows. The percent from 1992 to 2000 , from 2001 to 2009, and from 2010 to 2018 of HCV-HCC were $74.0 \%$ ( $n=311), 61.1 \%$ $(n=546)$, and $47.8 \%(n=414)$, respectively. Those of HBV-HCC were $14.2 \%(n=60), 19.0 \%(n=169)$, and 
$15.4 \%(n=134)$, respectively. Those of NBNC-HCC were $9 \%(n=38), 18.6 \%(n=166)$, and $35.7 \%(n=310)$, respectively. Those of BC-HCC were $2.4 \%(n=10), 0.8 \%(n=7)$, and $0.9 \%(n=8)$, respectively.

The number of NBNC-HCC has been increasing significantly, whereas rate of HCV-HCC patients tended to decrease, rate and number of NBNC-HCC tended to increase.

\section{Characteristics of patients with HCC based on etiology of liver diseases}

Characteristics of 2171 patients with HCC were shown in Table 1. Twenty-five patients with BC-HCC were excluded from the analysis because of the smaller number of patients. The patients with NBNC-HCC consisted of 417 males and 97 females. The median age was 72 years old, $64.2 \%$ had heavy alcohol consumption, $37.5 \%$ were positive for HBcAb. The median BMI was $24.3 \mathrm{~kg} / \mathrm{m}^{2}, 55.6 \%$ of patients had T2DM, 53.8\% had hypertension, and $48.0 \%$ had hyperlipidemia. The median platelet counts were $17.8 \times 10^{4} / \mu \mathrm{l}$, FIB-4 index were 3.89. ALBI grade were 231 were categorized as grade 1, 242 as grade 2, 41 as grade 3. On TNM staging for HCC according to the criteria of the Liver Cancer Study Group of Japan, 54 patients were categorized as stage I, 188 as stage II, 141 as stage III, and 131 as IV.

Patients with HBV-HCC consisted of 294 males and 67 females. The median age was 58 years old, $57.3 \%$ had heavy alcohol consumption, $100 \%$ were positive for HBcAb. The median BMI was $22.8 \mathrm{~kg} / \mathrm{m}^{2}, 20.2 \%$ of patients had T2DM, $22.7 \%$ of those had hypertension, and $29.0 \%$ of those had hyperlipidemia. The median platelet counts were $15.6 \times 10^{4} / \mu \mathrm{l}$ and FIB-4 index were 4.93 . ALBI grade were 176 were categorized as grade 1, 154 as grade 2,31 as grade 3 . On TNM staging for HCC, 61 patients were categorized as stage I, 103 as stage II, 90 as stage III, and 107 as IV.

Patients with HCV-HCC consisted of 841 males and 430 females. The median age was 68 years old, $44.2 \%$ had heavy alcohol consumption, and $31.2 \%$ were positive for HBcAb. The median BMI was 22.2 $\mathrm{kg} / \mathrm{m}^{2}, 29.3 \%$ of patients had T2DM $41.4 \%$ of those had hypertension, and $23.2 \%$ of those had hyperlipidemia. The median platelet counts were $11.7 \times 10^{4} / \mu$ l and FIB-4 index were 6.63 . ALBI grade were 453 were categorized as grade 1,741 as grade 2, 77 as grade 3 . On TNM staging for HCC, 332 patients were categorized as stage I, 467 as stage II, 321 as stage III, and 142 as IV.

Patients with NBNC-HCC were significantly older than other groups $(P<0.001)$. The complication rates of heavy alcohol consumption $(P<0.001)$, T2DM $(P<0.001)$, hypertension $(P<0.001)$, and hyperlipidemia $(P<0.001)$ in patients with NBNC-HCC were significantly higher than those in other groups. Furthermore, $\mathrm{BMI}$ and the platelet counts in NBNC-HCC patients were significantly higher than those in other groups $(P<0.001)$ and FIB-4 index in NBNC-HCC patients were significantly lower than those in other groups $(P<0.001)$. HCC stage in NBNC-HCC was significantly advanced than those in other groups (vs HBV-HCC; $P=0.006$, vs HCV-HCC; $P<0.001)$.

\section{Characteristics of patients with NBNC-HCC}


Previously, we reported up to 2009 [9], we compared with the characteristics of patients with NBNC-HCC from 2010 to 2018 by period (Table 2).

Patients with NBNC-HCC during 2010-2018 were significantly older than other groups $(P<0.001)$. As mentioned in the previous term, rate and number of NBNC-HCC tended to increase, accordingly, the number of patients with metabolic syndrome tended to increase, also.

Nineteen nine patients (48.5\%) were T2DM from 1992 to2009, 187 patients (60.3\%) from 2010 to 2018 $(P=0.008), 99$ patients (48.5\%) were hypertension from 1992 to 2000,178 patients $(57.4 \%)$ from 2010 to 2018 ( $P=0.046)$, and 80 patients (39.2\%) were hyperlipidemia from 1992 to 2000,167 patients $(53.8 \%)$ from 2010 to $2018(P=0.001)$, patients with metabolic syndrome increased significantly. On the other hands, the median FIB-4 index from 1992 to 2009 and from 2010 to 2018 were 4.37 and 3.61, respectively, were decreased $(P=0.026)$ in the last 10 years. Furthermore, the median platelet counts from 1992 to 2009 and from 2010 to 2018 were 15.1 and 17.9 , respectively, were higher $(P=0.013)$, also.

\section{Comparison of FIB-4 index by age in NBNC-HCC patients}

In addition, we assessed the FIB-4 index in NBNC-HCC patients by age. The median FIB-4 index indices in subjects from 20 to 39 years, from 40 to 59 years, from 60 to 79 years, and from 80 to 99 years were 1.43, $4.25,3.97$, and 4.39 , respectively. There was no significant difference in FIB-4 index for age stratification over 40 years. (Fig.2).

\section{Clinical characteristics of NBNC- HCC patients with hepatic resection}

Among 514 patients with primary NBNC-HCC, we studied clinical features of 194 patients who underwent hepatic resection (less number of autoimmune hepatitis or primary biliary cholangitis were excluded). We assessed the histological features of non-tumorous liver in NBNC-HCC patients. Here, in this study, we defined cases in which NASH or ALD could not clearly diagnosed in advance based on pathological results or medical history as cryptogenic.

Among NBNC-HCC patients, 29 (14.9\%) of those were NASH, 57 (29.3\%) of those were ALD, and 108 $(55.8 \%)$ of those were cryptogenic etiology.

The median age in cryptogenic group was significantly older than NASH groups $(P=0.018)$. The percentage of males was significantly higher in ALD and cryptogenic groups than in NASH group $(P<0.001)$.

The median platelet counts in cryptogenic group were significantly higher than in other groups (vs NASH; $P=0.025$, vs ALD; $P=0.023$ ) and FIB-4 index in cryptogenic group were significantly lower than in other groups (vs NASH; $P<0.001$, vs ALD; $P=0.002$ ). No significant differences in other clinical characteristics were observed (Table 3). 
The proportion of fibrosis stage $0,1,2,3$, and 4 were $0 \%, 6.8 \%, 10.4 \%, 10.4 \%$, and $72.4 \%$ in NASH patients, respectively. Those in ALD patients were 10.5\%, 15.8\%, 19.2\%, 12.2\%, and 38.6\%, respectively. Those in cryptogenic patients were $12.9 \%, 30.5 \%, 22.2 \%, 18.5 \%$ and $15.7 \%$, respectively. Cirrhosis was found in $72.4 \%, 38.6 \%$, and $15.5 \%$ of patients with NASH, ALD and cryptogenic, respectively. The prevalence of cirrhosis in NASH were significantly higher than other groups $(P<0.001)$ (Fig. 3$)$.

\section{Discussion}

In the present study, we retrospectively assessed the clinical characteristics of HCC. The rate and the number of NBNC-HCC tended to increase, whereas those of HCV-HCC tended to decrease especially in recent years. Five hundred and fourteen patients (23.6\%) of HCC were NBNC-HCC in 1992-2018. Patients with NBNC-HCC were significantly older. The rates of heavy alcohol consumption, T2DM, hypertension, and hyperlipidemia were significantly higher than those in other groups. Furthermore, in NBNC-HCC patients, BMI and the platelet counts were significantly higher and FIB-4 index were significantly lower than other groups.

In accordance with previously reported findings, our findings were in agreement with those that people with obesity, T2DM or heavy alcohol consumption and metabolic syndrome are at an increased risk of developing HCC $[45,46]$. As previously reported [8], these complications are likely to become HCC risks in the future and need attention.

As shown in Table 2, the patients with NBNC-HCC during 2010-2018 were significantly older, and the rates of T2DM, hypertension, and hyperlipidemia were higher than those from 1992 to 2009 . The increase in metabolic syndrome is suggested to affect the increase of NBNC-HCC. Furthermore, the platelet counts were higher and FIB-4 index were lower than those from 1992 to 2009 . This might be because HCC occurred even in patients with non-advanced liver fibrosis. These observation shows us that NBNC patients need to be aware that HCC develops independently of fibrosis stage. Although it is suggested that the systemic metabolic syndrome environment may cause a systemic inflammatory condition and this might be associated with thrombocytosis, the reason why platelets are maintained more than before has not been clarified in this study, and awaits future study. FIB-4 index tended to be higher in the elderly, but there is no significant difference by age as shown in Fig.2. Because of small number of young patients with HCC, it is unsure but FIB-4 index might become a useful screening index of HCC.

The TNM stage in NBNC-HCC was significantly advanced more than that in other groups. This is because patients with NBNC-HCC do not receive regular HCC surveillance whereas HBV-HCC and HCV-HCC patients received regular HCC surveillance. HBV and HCV infection related to long-term infection and the progression of liver fibrosis often leads to chronic, hepatitis, cirrhosis, cirrhosis-related complications, and HCC [47], therefore, HBV and HCV patients received regular surveillance. Similar results have been reported in our past reports [8-10], it is necessary to recommend to receive regular HCC surveillance using ultrasonography and/or other available modalities even if patients don't have liver disease such as viral hepatitis, since still so many NBNC-HCC is detected in an advanced stage. Moreover, it is also important 
to perform screening using $\mathrm{CT}$ and MRI with new imaging and scoring system, we think it is necessary to diagnose HCC earlier. $[48,49]$. Therefore, in order to diagnose HCC at an early stage, it is necessary to use FIB-4 index together.

From the examination of histologically diagnosed background liver, cirrhosis was detected in $72.4 \%$, $38.6 \%$, and $15.5 \%$ of patients with NASH, ALD and cryptogenic, respectively. The prevalence of cirrhosis in $\mathrm{NASH}$ were significantly higher than that in other groups, and cryptogenic had a lower rate of advanced fibrosis. Until now, many studies have reported that NASH can progress to cirrhosis [50-53], the severity of liver fibrosis must be estimated to determine the surveillance and for treatment of NAFLD. In patients with cryptogenic, platelet counts tended to be higher, FIB-4 index was lower, and fibrosis was not severe. At present, FIB-4 index, one of the recommended scoring systems, uses a cutoff value of 2.67 as a progression of fibrosis. The median FIB-4 index in cryptogenic was 2.56 and this is similar to the value that has been regarded as an index that increases the risk of developing HCC [54,55]. From these results as well, the FIB-4 index could contribute to HCC screening.

In our study, patients with cryptogenic HCC accounted for the majority of NBNC-HCC. Most (93.5\%) of those in cryptogenic patients had any of T2DM, hypertension, hyperlipidemia, obesity, and HBc antibody. Considering the above, it is possible that HCC progressed without being diagnosed with NASH or Alcoholic liver disease during the course. In the future, patients with metabolic syndrome will need to pay attention to HCC development.

Limitations of our study are that not all the pathological aspects of background liver were available in NBNC-HCC group and that the study population may not reflect the entire patients with HCC in Japan.

\section{Conclusions}

The present study elucidated that NBNC-HCC has gradually been increasing in recent years culminating in $40 \%$ of all HCC patients. The presence of metabolic syndrome were important risk factors for NBNC-HCC. Furthermore, only advanced fibrosis in NBNC-HCC are not the determinant for increased HCC risk, and HCC from non-cirrhosis are not uncommon. However, FIB-4 index tends to be higher, considered as one of useful screening of HCC.

\section{Abbreviations}

HCC: Hepatocellular carcinoma; HBV: Hepatitis B virus; HCV: Hepatitis C virus; NASH: Non-alcoholic steatohepatitis; ALD Alcoholic liver diseas

\section{Declarations}

\section{Acknowledgements}

Not applicable 


\section{Authors' contributions}

The involvement of each author was as follows: YN (1-5); HH(2); YA(2); YK(2); SU (2); YNishida(2); YT(2); KM(2); HF(2); AO (2); TN (2); EM (2); MY(2); WO(2); TK(2); ST (2); AH(2); DM (2); MT (2); MI(2); STakahashi(2); KC (1, 2, 6, 7); $\mathrm{AH}(1,2,6,7)$

Key: (1) Conception and design; (2) Acquisition of data; (3) Analysis and interpretation of data; (4) Drafting of the manuscript; (5) Statistical analysis; (6) Study supervision; (7) Critical revision of the manuscript. All authors read the final version of this article and approved its content and submission for publication. All authors agreed to be accountable for all aspects of the work in ensuring that questions related to the accuracy or integrity of any part of the work are appropriately investigated and resolved.

\section{Funding}

The funders had no role in study design, data collection and analysis, decision to publish, or preparation of the manuscript. No additional external funding was received for this study.

\section{Availability of data and materials}

All data generated or analysed during this study are included in this published article.

\section{Ethics approval and consent to participate}

All participants provided written informed consent to participate in the study according to the process approved by the ethical committee of Hiroshima University and conforming to the ethical guidelines of the Declaration of Helsinki.

\section{Consent for publication}

Not applicable. The authors declare that no patient data (details, images or videos relating to individual participants) are included in this article.

\section{Competing interests}

Kazuaki Chayama has received honoraria from Bristol-Myers Squibb, MSD K.K., AbbVie Inc., Gilead Sciences and Dainippon Sumitomo Pharma and research funding from Dainippon Sumitomo Pharma, MSD K. K and AbbVie Inc..

Hiroshi Aikata has received honoraria from Bayer Yakuhin, Ltd and Eisai.

\section{Author details}


${ }^{1}$ Department of Gastroenterology and Metabolism, Graduate School of Biomedical and Health Science, Hiroshima University, Hiroshima, Japan. ${ }^{2}$ Research Center for Hepatology and Gastroenterology, Hiroshima University, Hiroshima, Japan. ${ }^{3}$ Cancer Treatment Center, Hiroshima University Hospital. ${ }^{4}$ Natural Science Center for Basic Research and Development, Hiroshima University, Hiroshima, Japan. ${ }^{5}$ Institute of Physical and Chemical Research (RIKEN) Center for Integrative Medical Sciences, Yokohama, Japan

\section{References}

1. Vital Statistics Japan (Ministry of Health, Labour and Welfare) 2018

2. Kudo M, Izumi N, Kubo S, Kokudo N, Sakamoto M, Shiina S, et al. Report of the 20th Nationwide follow-up survey of primary liver cancer in Japan Hepatology Res. 2020; 50: 15-46.

3. de Martel C, Maucort-Boulch D, Plummer M, Franceschi S. World-wide relative contribution of hepatitis B and C viruses in hepatocellular carcinoma. Hepatology. 2015; 62:1190-1200.

4. Bertuccio P, Turati F, Carioli G, Rodriguez T, La Vecchia C, Malvezzi M, et al. Global trends and predictions in hepatocellular carcinoma mortality. J Hepatol. 2017; 67:302-309.

5. Liaw YF, Sung JJ, Chow WC, Farrell G, Lee CZ, Yuen H, et al. Lamivudine for patients with chronic hepatitis B and advanced liver disease. N Engl J Med. 2004; 351:1521-1531.

6. Yokosuka O, Takaguchi K, Fujioka S, Shindo M, Chayama K, Kobashi H, et al. Long-term use of entecavir in nucleoside-naïve Japanese patients with chronic hepatitis B infection. J Hepatol. 2010; 52:791-799.

7. Kobayashi M, Suzuki F, Fujiyama S et al. Sustained virologic response by direct antiviral agents reduces the incidence of hepatocellular carcinoma in patients with HCV infection. J Med Virol 2017; 89: 476-83. 
8. Nagaoki Y, Hyogo H, Aikata H, Tanaka M, Naeshiro N, Nakahara T, et al. Recent trend of clinical features in patients with hepatocellular carcinoma. Hepatology Res. 2012; 42: 368-375.

9. Tateishi R, Okanoue T, Fujiwara N, Okita K, Kiyosawa K, Omata M, et al. Clinical characteristics, treatment, and prognosis of non- $\mathrm{B}$, non-C hepatocellular carcinoma: a large retrospective multicenter cohort study. J Gastroenterol. 2015; 50:350-360.

10. Tateishi R, Uchino K, Fujiwara N, Takehara T, Okanoue T, Seike M, et al. A nationwide survey on non-B, non-C hepatocellular carcinoma in Japan: 2011-2015 update. J Gastroenterol; 2019: 54:367-376.

11. Singal AK, Bataller R, Ahn J, Kamath PS, Vijay H, Shah VH. ACG Clinical Guideline: Alcoholic Liver Disease. Am J Gastroenterol. 2018; 113: 175-194.

12. Morgan TR, Mandayam S, Jamal MM. Alcohol and hepatocellular carcinoma. Gastroenterology 2004; 127: 87-S96.

13. Szabo G, Kamath PS, Shah VH, Thursz M, Mathurin P. Alcohol-Related Liver Disease: Areas of Consensus, Unmet Needs and Opportunities for Further Study. Hepatology, 2019; 69: 2271-2283.

14. Starley BQ, Calcagno CJ, Harrison SA. Nonalcoholic fatty liver disease and hepatocellular carcinoma: a weighty connection. Hepatology. 2010; 51:1820-1832.

15. Hashimoto E, Tokushige K. Hepatocellular carcinoma in non-alcoholic steatohepatitis: growing evidence of an epidemic? Hepatol Res. 2012; 42:1-14.

16. Ekstedt M, Hagstrom H, Nasr P. Fibrosis Stage Is the Strongest Predictor for Disease-Specific Mortality in NAFLD After Up to 33 Years of Follow-Up. Hepatology 2015; 61:1547-1554. 
17. Welzel TM, Graubard B, Quraishi S, Zeuzem S, Davila JA, El-Serag HB, et al. Population-attributable fractions of risk factors for hepatocellular carcinoma in the United States. Am J Gastroenterol. 2013; 108:1314-1321.

18. Bhaskaran K, Douglas I, Forbes H, dos-Santos-Silva I, Leon DA, Smeeth L. Body-mass index and risk of 22 specific cancers: a population-based cohort study of 5.24 million UK adults. Lancet. 2014; 384:755-765.

19. Streba LAM, Vere CC, Rogoveanu I, CT.Nonalcoholic fatty liver disease, metabolic risk factors, and hepatocellular carcinoma: An open questionWorld J Gastroenterol 2015; 14; 21: 4103-4110

20. Noureddin M, Rinella ME. Nonalcoholic Fatty Liver Disease, Diabetes, Obesity, and Hepatocellular CarcinomaClin Liver Dis. 2015; 19: 361-379.

21. Tanaka K, Tsuji I, Tamakoshi A, Matsuo K, Ito H, Wakai K,Nagata C, Mizoue T, Sasazuki S, Inoue M, Tsugane S. Obesity and liver cancer risk: an evaluation based on a systematic review of epidemiologic evidence among the Japanese population. Jpn J Clin Oncol 2012; 42: 212-221.

22. El-Serag HB, Rudolph KL. Hepatocellular carcinoma: epidemiology and molecular carcinogenesis. Gastroenterology. 2007; 132:2557-2576.

23. Hung $\mathrm{CH}$, Wang JH, Hu TH, Chen $\mathrm{CH}$, Chang KC, Yen YH, Kuo YH, Tsai MC, Lu SN, Lee CM. Insulin resistance is associated with hepatocellular carcinoma in chronic hepatitis $\mathrm{C}$ infection. World $\mathrm{J}$ Gastroenterol 2010; 16: 2265-2271.

24. Giovannucci E, Harlan DM, Archer MC, Bergenstal RM, Gapstur SM, Habel LA, et al. Diabetes and cancer: a consensus report. CA Cancer J Clin. 2010; 60:207-221. 
25. Rao Kondapally Seshasai S, Kaptoge S, Thompson A, Di Angelantonio E, Gao P, Sarwar N, et al. Diabetes mellitus, fasting glucose, and risk of cause-specific death. N Engl J Med. 2011;364:829841.

26. Siegel AB, Zhu AX. Metabolic syndrome and hepatocellular carcinoma: two growing epidemics with a potential link. Cancer. 2009;115:5651-5661.

27. Shoelson SE, Herrero L, Naaz A. Obesity, inflammation, and insulin resistance. Gastroenterology. 2007;132:2169-2180.

28. Stickel F, Hellerbrand C. Non-alcoholic fatty liver disease as a risk factor for hepatocellular carcinoma: mechanisms and implications. Gut. 2010;59:1303-1307.

29. Park EJ, Lee JH, Yu GY, et al. Dietary and genetic obesity promote liver inflammation and tumorigenesis by enhancing IL-6 and TNF expression. Cell. 2010;140:197-208.

30. Hashimoto E, Tokushige K, Ludwig J. Diagnosis and classification of non-alcoholic fatty liver disease and non-alcoholic steatohepatitis: Current concepts and remaining challenges. Hepatology Res 2015; 45: 20-28.

31. Estes C, Razavi H, Loomba R, Younossi Z, Sanyal AJ. Modeling the Epidemic of Nonalcoholic Fatty Liver Disease Demonstrates an Exponential Increase in Burden of Disease. Hepatology 2018; 67:123-133.

32. Wong RJ, Aguilar M, Cheung R, Perumpail RB, Harrison SA, Younossi ZM, et al. Nonalcoholic steatohepatitis is the second leading etiology of liver disease among adults awaiting liver transplantation in the United States. Gastroenterology 2015; 148:547-555. 
33. Tokushige $\mathrm{K}$, Hyogo H, Nakajima T, Ono M, Kawaguchi T, Honda K, et al. Hepatocellular carcinoma in Japanese patients with nonalcoholic fatty liver disease and alcoholic liver disease: multicenter survey. J Gastroenterol 2016; 51:586-596.

34. Cholankeril G, Ahmed A. Alcoholic Liver Disease Replaces Hepatitis C Virus Infection as the Leading Indication for Liver Transplantation in the United States. Clinical Gastroenterology and Hepatology $2018 ; 16: 1356-1358$.

35. Pais R, Lebray P, Rousseau G, Charlotte F, Esselma G, Savier E, et al. Nonalcoholic fatty liver disease increases the risk of hepatocellular carcinoma in patients with alcohol-associated cirrhosis awaiting liver transplants. Clin Gastroenterol Hepatol. 2015; 135:992-999.

36. Setiawan VW, Stram DO, Porcel J. revalence of chronic liver disease and cirrhosis by underlying cause in understudied ethnic groups: The multiethnic cohort. 2016; 4:1969-1977.

37. Shah AG, Lydecker A, Murray K, Tetri BN, Contos MJ, Sanyal AJ, et al. Comparison of noninvasive markers of fibrosis in patients with nonalcoholic fatty liver disease. Clin Gastroenterol Hepatol. 2009; 7: 1104-1112.

38. Johnson PJ, Berhane S, Kagebayashi C, Satomura S, Teng M, Reeves HL, et al: Assessment of liver function in patients with hepatocellular carcinoma: a new evidence-based approach-the ALBI grade. J Clin Oncol 2015; 33: 550-558.

39. Japanese Society for Biomedical Research on Alcohol. Japanese Society for Biomedical Research on Alcohol Diagnostic Criteria for Alcoholic Liver Disease 2011 Edition.

40. The Committee of Japan Diabetes Society on the Diagnostic Criteria of Diabetes Mellitus. Report of the Committee on the classification and diagnostic criteria of diabetes mellitus. J Diabetes Invest 2010; 1: 212-228. 
41. Brunt EM, Janney CG, Di Biscegli AM, Neuschwander-Tetri BA, Bacon BR. Nonalcoholic steatohepatitis: a proposal for grading and staging the histological lesions. Am J Gastroenterol.1999; 94: 2467-2474.

42. Brunt EM, Kleiner DE, Wilson LA, et al. NASH Clinical Research Network (CRN). Nonalcoholic fatty liver disease (NAFLD) activity score and the histopathologic diagnosis in NAFLD: distinct clinicopathologic meanings. Hepatology. 2011; 53: 810-20.

43. Kokudo N, Takemura N, Hasegawa K,et al.Clinical practice guidelines for hepatocellular carcinoma: The Japan Society of Hepatology 2017 (4th JSH-HCC guidelines) 2019 update. Hepatol Res. 2019; 49:1109-1113.

44. Liver Cancer Study Group of Japan. General rules for the clinical and pathological study of primary liver cancer, 6th. Englished. Tokyo: Kasahara; 2019.

45. Shima T, Uto H, Ueki K, Kohgo Y, Yasui K, Nakamura N, et al. Hepatocellular carcinoma as a leading cause of cancer-related deaths in Japanese type 2 diabetes mellitus patient. J Gastroenterol 2019; 54:64-77.

46. Tobari M, Hashimoto E. Characteristic Features of Nonalcoholic Fatty Liver Disease in Japan with a Focus on the Roles of Age, Sex and Body Mass Index. Gut Liver, Published online January 3, 2020.

47. Besheer T, Elbendary M, Elalfy H, El-Maksoud MA, Salah M, Zalata K, et al. Prediction of Fibrosis Progression Rate in Patients with Chronic Hepatitis C Genotype 4: Role of Cirrhosis Risk Score and Host Factors. J Interferon Cytokine Research 2017;37:97-102.

48. Abdel Razek AAK, El-Serougy LG, Saleh GA, Shabana W, Abd El-Wahab R. Liver Imaging Reporting and Data System Version 2018: What Radiologists Need to Know. J Comput Assist Tomogr 2020; 44: 168-177. 
49. Abdel Razek AAK, El-Serougy LG, Saleh GA, Abd el-wahab R, Shabana W. Interobserver Agreement of Magnetic Resonance Imaging of Liver Imaging Reporting and Data System Version 2018. J Comput Assist Tomogr 2020; 44: 118-123.

50. Estes C, Anstee QM, Arias-Loste MT, Bantel H, Bellentani S, Caballeria J, et al. Modeling NAFLD disease burden in China, France, Germany, Italy, Japan, Spain, United Kingdom, and United States for the period 2016-2030. J Hepatol. 2018; 69:896-904.

51. Dulai PS, Singh S, Patel J, Soni M, Prokop LJ, Younossi Z, et al. Increased risk of mortality by fibrosis stage in nonalcoholic fatty liver disease: Systematic review and meta-analysis. 2017; 65:1557-1565.

52. Angulo P, Kleiner DE, Dam-Larsen S. Liver Fibrosis, but No Other Histologic Features, Is Associated With Long-term Outcomes of Patients With Nonalcoholic Fatty Liver Disease. 2015; 149:389-397.

53. Hashimoto E, Taniai, Tokushige K. Characteristics and diagnosis of NAFLD/NASH. Journal of Gastroenterology and Hepatology. 2013; 28: 64-70.

54. Vallet-Pichard A, Mallet V, Nalpas B, Verkarre V, Nalpas A, Dhalluin-Venier V, et al. FIB-4: an inexpensive and accurate marker of fibrosis in $\mathrm{HCV}$ infection comparison with liver biopsy and fibrotest. Hepatology. 2007; 46: 32-36.

55. Sumida Y, Yoneda M, Hyogo H, Itoh Y, Ono M, Fujii H, et al. Validation of the FIB4 index in a Japanese nonalcoholic fatty liver disease population. BMC Gastroenterol. 2012; 12: 2. doi: 10.1186/1471.

\section{Tables}

Table1 Characteristics of patients with HCC based on etiology of liver disease 


\begin{tabular}{|c|c|c|c|c|c|}
\hline & & & & $P$ value & \\
\hline & NBNC & HBV & $\mathrm{HCV}$ & NBNC & NBNC \\
\hline & $n=514$ & $n=361$ & $n=1271$ & vs.HBV & vs.HCV \\
\hline Age (years) & $72(21-90)$ & $58(31-80)$ & $68(28-84)$ & $<0.001$ & $<0.001$ \\
\hline $\begin{array}{l}\text { Gender } \\
\text { (male/female) }\end{array}$ & $417 / 97$ & $294 / 67$ & $841 / 430$ & N.S & $<0.001$ \\
\hline $\begin{array}{l}\text { Alcohol } \\
\text { consumption }\end{array}$ & $330(64.2 \%)$ & $207(57.3 \%)$ & $562(44.2 \%)$ & 0.024 & $<0.001$ \\
\hline $\begin{array}{l}\text { Antibody to } \\
\text { hepatitis B core } \\
\text { antigen }\end{array}$ & $179(34.8 \%)$ & $361(100 \%)$ & $397(31.2 \%)$ & $<0.001$ & $<0.001$ \\
\hline $\begin{array}{l}\text { Body mass index } \\
\left(\mathrm{kg} / \mathrm{m}^{2}\right)\end{array}$ & 24.3(14.3-41.2) & 22.8(14.4-31.7) & $22.2(14.8-33.6)$ & $<0.001$ & $<0.001$ \\
\hline Diabetes mellitus & $286(55.6 \%)$ & $73(20.2 \%)$ & $373(29.3 \%)$ & $<0.001$ & $<0.001$ \\
\hline Hypertension & $277(53.8 \%)$ & $82(22.7 \%)$ & $527(41.4 \%)$ & $<0.001$ & $<0.001$ \\
\hline Hyperlipidemia & $247(48.0 \%)$ & $105(29.0 \%)$ & $295(23.2 \%)$ & $<0.001$ & $<0.001$ \\
\hline $\operatorname{ALT}(\mathrm{IU} / \mathrm{I})$ & $47(6-435)$ & $55(10-644)$ & $51(9-847)$ & $<0.001$ & $<0.001$ \\
\hline $\begin{array}{l}\text { Platelet counts } \\
\left(\times 10^{4} / \mu \mathrm{l}, \text { median }\right)\end{array}$ & $17.8(2.7-57.3)$ & 15.6(1.9-38.1) & 11.7(1.1-28.1) & $<0.001$ & $<0.001$ \\
\hline FIB-4 index & $3.89(0.25-28.31)$ & $\begin{array}{l}4.93(0.35- \\
34.69)\end{array}$ & 6.63(0.69-39.61) & $<0.001$ & $<0.001$ \\
\hline ALBI grade $1 / 2 / 3$ & 231/242/41 & $176 / 154 / 31$ & $453 / 741 / 77$ & N.S & $<0.001$ \\
\hline $\begin{array}{l}\text { Child Pugh grade } \\
\text { A/B/C }\end{array}$ & $382 / 105 / 27$ & $267 / 71 / 23$ & $919 / 304 / 48$ & N.S & N.S \\
\hline $\begin{array}{l}\text { Antinuclear } \\
\text { antibody }\end{array}$ & 68(13.2\%) & $46(12.7 \%)$ & $188(14.9 \%)$ & N.S & N.S \\
\hline $\begin{array}{l}\text { Anti-mitochondrial } \\
\text { antibodies }\end{array}$ & $15(2.9 \%)$ & $3(0.8 \%)$ & $20(1.6 \%)$ & 0.032 & N.S \\
\hline $\begin{array}{l}\text { HCC stage * } \\
(\mathrm{I} / \mathrm{II} / \mathrm{III} / \mathrm{IV})\end{array}$ & $54 / 188 / 141 / 131$ & 61/103/90/107 & $332 / 467 / 321 / 142$ & 0.006 & $<0.001$ \\
\hline
\end{tabular}

Continuous data are represented as median and range, and categorical data are represented as numbers of patients.

*;determined according to the criteria set by the Liver Cancer Study Group of Japan; 
HBV, hepatitis B virus; HCV, hepatitis C virus; NBNC, negative for hepatitis B surface antigen and antibody to hepatitis $C$ virus; FIB-4, fibrosis-4; ALBI grade, albumin-bilirubin grade; NS, not significant.

Table2 Characteristics of patients with NBNC-HCC by period

\begin{tabular}{|llll|}
\hline & & & P value \\
& $1992-2009$ & $2010-2018$ & $2010-2018$ \\
& & & vs \\
\hline Age (years) & $\mathrm{n}=204$ & $\mathrm{n}=310$ & $1992-2009$ \\
\hline Gender (male/female) & $67(21-85)$ & $72(22-90)$ & $<0.001$ \\
\hline Alcohol consumption & $163 / 41$ & $254 / 56$ & N.S \\
\hline Antibody to hepatitis B core antigen & $83(40.6 \%)$ & $96(30.9 \%)$ & 0.023 \\
\hline Body mass index (kg/m $\left.{ }^{2}\right)$ & $23.8(14.3-38.9)$ & $23.9(15.3-41.2)$ & N.S \\
\hline Diabetes mellitus & $99(48.5 \%)$ & $187(60.3 \%)$ & 0.008 \\
\hline Hypertension & $99(48.5 \%)$ & $178(57.4 \%)$ & 0.046 \\
\hline Hyperlipidemia & $80(39.2 \%)$ & $167(53.8 \%)$ & 0.001 \\
\hline ALT(IU/I) & $47(8-435)$ & $48(6-388)$ & N.S \\
\hline Platelet counts(x10 $4 / \mu$ l, median) & $15.1(3.5-49.1)$ & $17.9(2.7-57.3)$ & 0.013 \\
\hline FIB-4 index & $4.37(0.67-28.31)$ & $3.61(0.25-22.34)$ & 0.026 \\
\hline ALBI grade 1/2/3 & $93 / 96 / 15$ & $138 / 146 / 26$ & N.S \\
\hline Child Pugh grade A/B/C & $151 / 42 / 11$ & $231 / 63 / 16$ & N.S \\
\hline Antinuclear antibody & $23(11.2 \%)$ & $45(14.5 \%)$ & N.S \\
\hline Anti-mitochondrial antibodies & $9(4.4 \%)$ & $6(1.9 \%)$ & N.S \\
\hline
\end{tabular}

Continuous data are represented as median and range, and categorical data are represented as numbers of patients.

*;determined according to the criteria set by the Liver Cancer Study Group of Japan; 
HBV, hepatitis B virus; HCV, hepatitis C virus; NBNC, negative for hepatitis B surface antigen and antibody to hepatitis $C$ virus; FIB-4, fibrosis-4; ALBI grade, albumin-bilirubin grade; NS, not significant.

Table3 Clinical characteristics of NBNC- HCC patients with Hepatic resection 
$P$ value

\begin{tabular}{|c|c|c|c|c|c|c|}
\hline & $\mathrm{NASH}$ & $\begin{array}{l}\text { Alcoholic } \\
\text { liver } \\
\text { disease }\end{array}$ & $\begin{array}{l}\text { Cryptogenic } \\
\text { etiology }\end{array}$ & $\begin{array}{l}\text { NASH } \\
\text { vs }\end{array}$ & $\begin{array}{l}\text { NASH } \\
\text { vs }\end{array}$ & $\begin{array}{l}\text { Alcoholic } \\
\text { liver } \\
\text { disease } \\
\text { vs }\end{array}$ \\
\hline & $n=29$ & $n=57$ & $n=108$ & $\begin{array}{l}\text { Alcoholic } \\
\text { liver } \\
\text { disease }\end{array}$ & $\begin{array}{l}\text { Cryptogenic } \\
\text { etiology }\end{array}$ & $\begin{array}{l}\text { Cryptogenic } \\
\text { etiology }\end{array}$ \\
\hline Age (years) & $66(47-82)$ & 71(54-88) & $73(25-85)$ & 0.040 & 0.018 & N.S \\
\hline $\begin{array}{l}\text { Gender } \\
\text { (male/female) }\end{array}$ & $14 / 15$ & $55 / 2$ & $84 / 21$ & $<0.001$ & $<0.001$ & 0.004 \\
\hline $\begin{array}{l}\text { Body mass } \\
\text { index }\left(\mathrm{kg} / \mathrm{m}^{2}\right)\end{array}$ & $\begin{array}{l}24.4(17.4- \\
31.3)\end{array}$ & $\begin{array}{l}23.6(15.2- \\
32.4)\end{array}$ & $\begin{array}{l}23.5(16.0- \\
34.2)\end{array}$ & N.S & N.S & N.S \\
\hline $\begin{array}{l}\text { Antibody to } \\
\text { hepatitis B core } \\
\text { antigen }\end{array}$ & $10(34.4 \%)$ & $17(29.8 \%)$ & $34(31.4 \%)$ & N.S & N.S & N.S \\
\hline $\begin{array}{l}\text { Diabetes } \\
\text { mellitus }\end{array}$ & $13(44.8 \%)$ & $30(54.3 \%)$ & $64(59.2 \%)$ & N.S & N.S & N.S \\
\hline Hypertension & 17(58.6\%) & $34(59.6 \%)$ & $71(65.7 \%)$ & N.S & N.S & N.S \\
\hline Hyperlipidemia & $8(25.5 \%)$ & $15(26.3 \%)$ & $36(33.3 \%)$ & N.S & N.S & N.S \\
\hline AST (IU/I) & $\begin{array}{l}53(19- \\
397)\end{array}$ & $\begin{array}{l}56(19- \\
146)\end{array}$ & $39(15-308)$ & N.S & 0.041 & 0.035 \\
\hline ALT (IU/L) & $\begin{array}{l}58(17- \\
435)\end{array}$ & $\begin{array}{l}45(11- \\
175)\end{array}$ & $38(8-340)$ & 0.035 & 0.032 & 0.043 \\
\hline Y-GTP (IU/L) & $\begin{array}{l}74(21- \\
612)\end{array}$ & $\begin{array}{l}82(18- \\
836)\end{array}$ & $70(8-583)$ & N.S & N.S & N.S \\
\hline $\begin{array}{l}\text { Platelet counts } \\
\left(\times 10^{4} / \mu \mathrm{l}\right)\end{array}$ & $\begin{array}{l}15.4(7- \\
23.6)\end{array}$ & $\begin{array}{l}15.1(5.2- \\
29.1)\end{array}$ & $\begin{array}{l}18.4(6.1- \\
43.3)\end{array}$ & N.S & 0.025 & 0.023 \\
\hline FIB-4 index & $\begin{array}{l}3.68(1.03- \\
17.52)\end{array}$ & $\begin{array}{l}3.28(1.40- \\
11.37)\end{array}$ & $\begin{array}{l}2.56(0.47- \\
8.66)\end{array}$ & N.S & $<0.001$ & 0.002 \\
\hline $\begin{array}{l}\text { ALBI grade } \\
1 / 2 / 3\end{array}$ & $17 / 9 / 3$ & $38 / 17 / 2$ & $74 / 32 / 2$ & N.S & N.S & N.S \\
\hline
\end{tabular}

Continuous data are represented as median and range, and categorical data are represented as numbers of patients.

AST, aspartate aminotransferase; ALT, alanine aminotransferase; 
Y-GTP, y-glutamytranspeptidase; NASH, non-alcoholic steatohepatitis; FIB-4, fibrosis-4; ALBI grade, albumin-bilirubin grade; NS, not significant.

\section{Figures}

Fig. 1

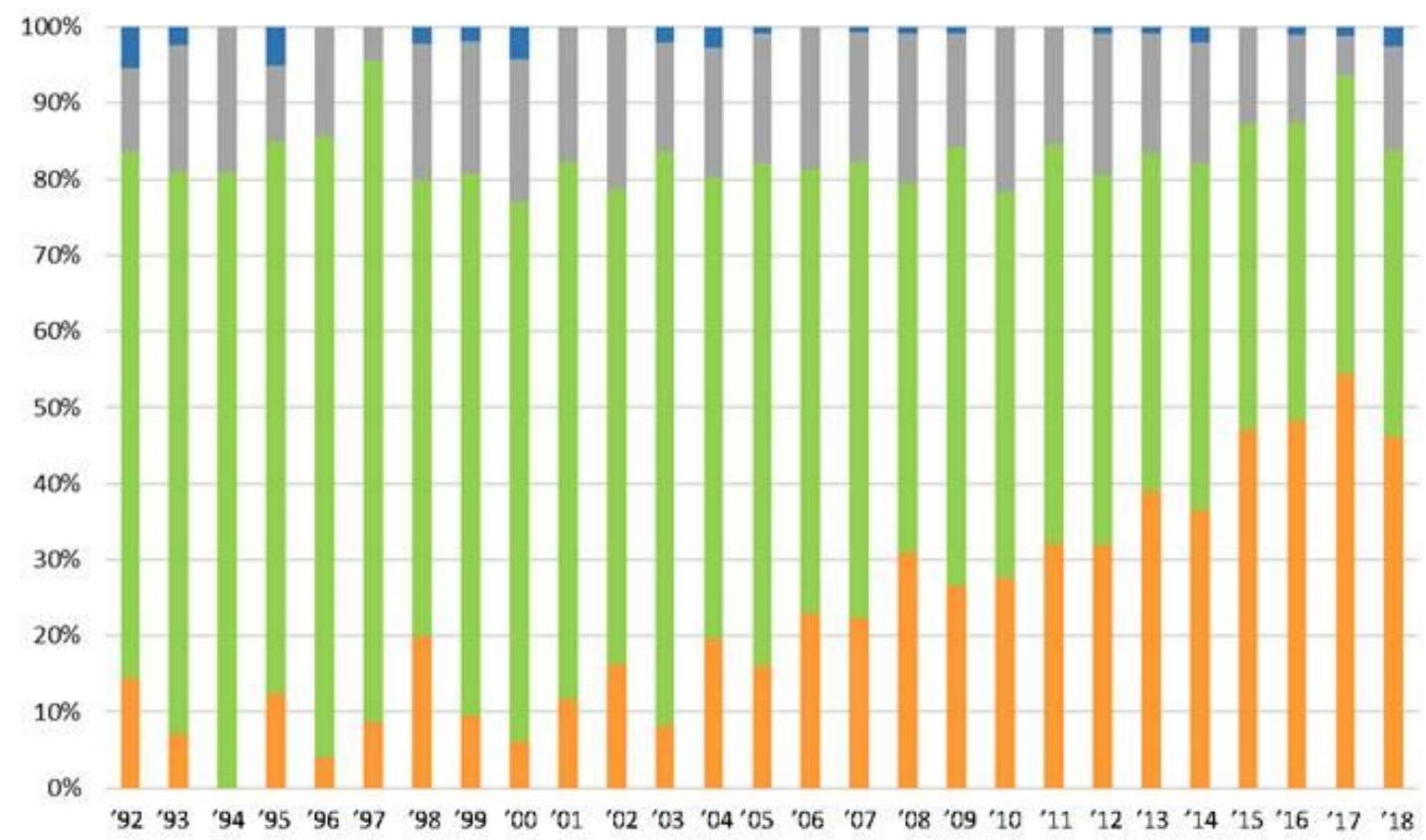

\section{Figure 1}

Trend of etiology of liver diseases in patients with HCC We show that distribution of trend of etiology of liver disease in patients with HCC of every 8 years, among 2171 patients from 1992 to 2018 . The line graph shows the actual number of NBNC-HCC. $\square: N B N C ; \square: H C V ; \square: H B V ; \square: B+C$. 
Fig. 2

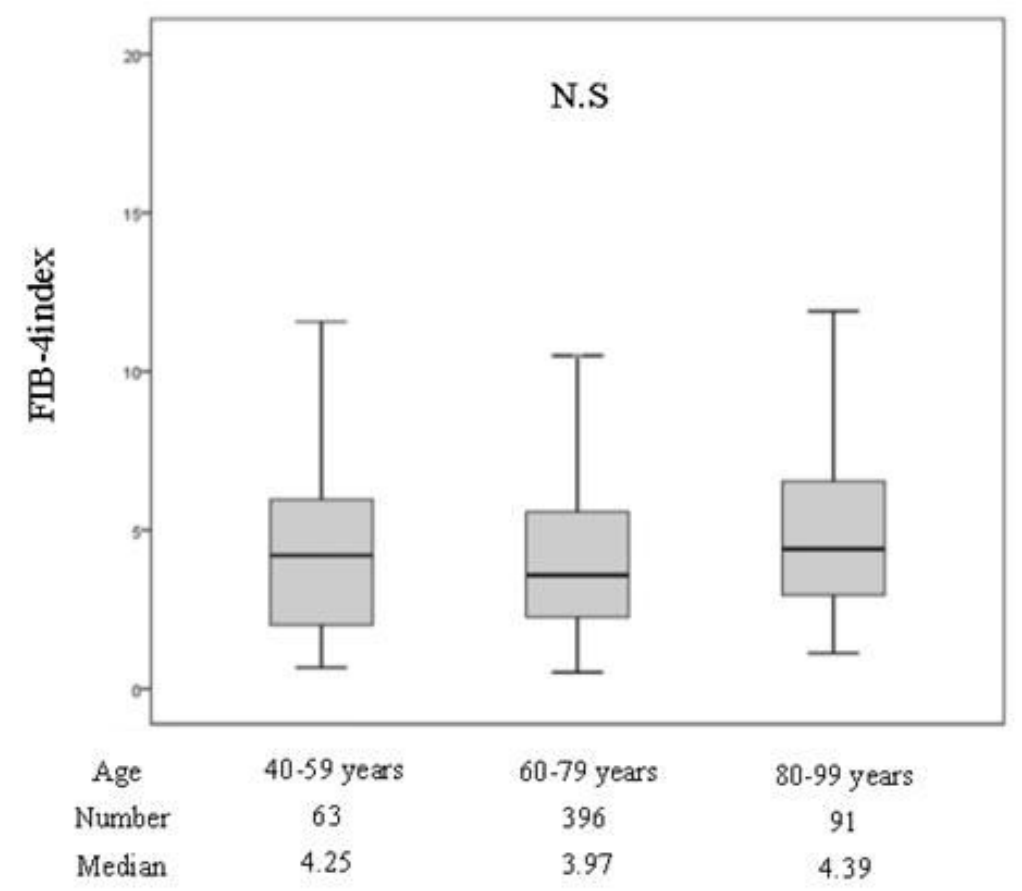

Figure 2

We assessed the FIB-4 index in NBNC-HCC patients by age. In these box-and-whisker plots, lines within the boxes represent median values; the upper and lower lines of the boxes represent the 75th and 25th percentiles, respectively; the upper and lower bars outside the boxes represent the 90th and 10th percentiles, respectively. NS, not significant 
Fig. 3

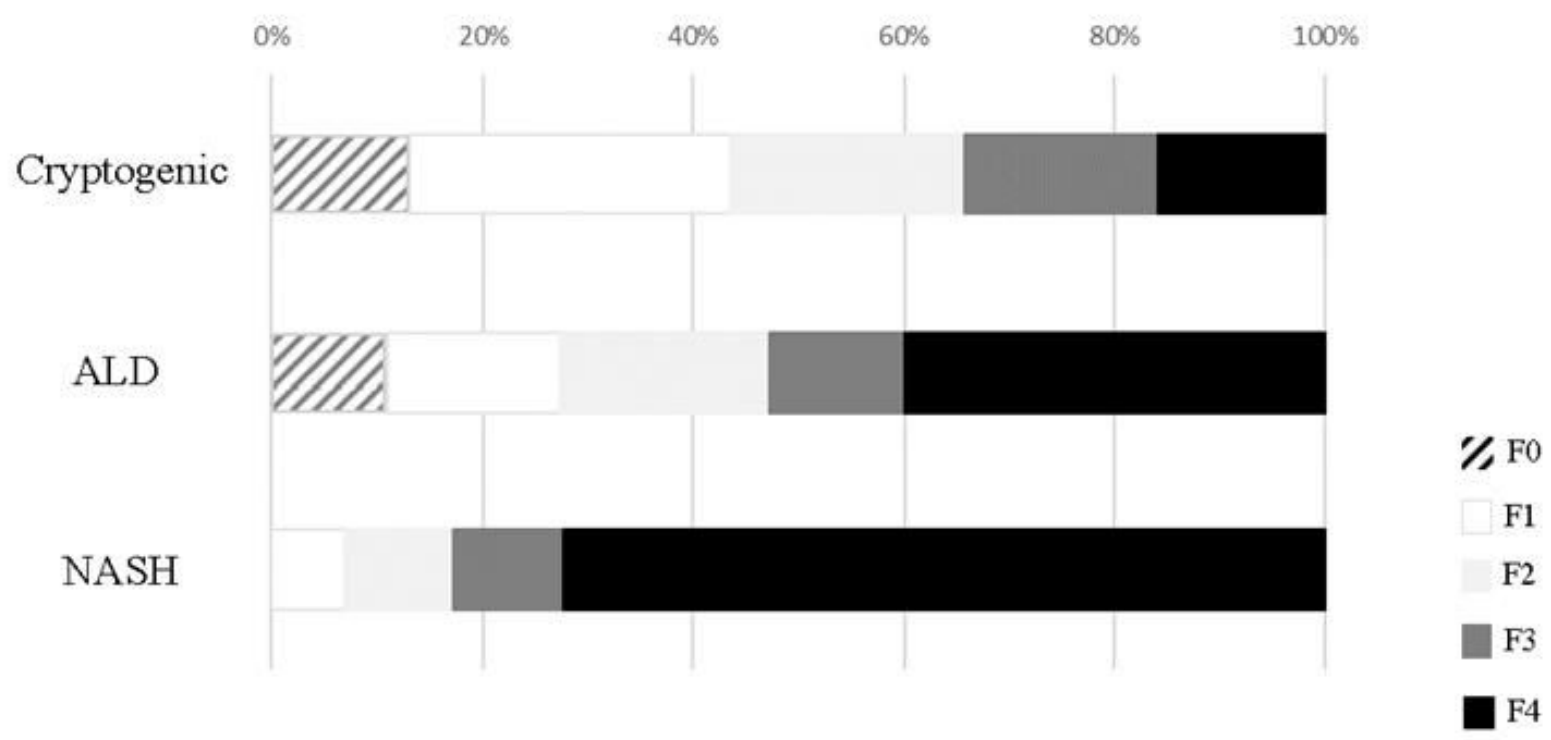

\section{Figure 3}

The proportion of fibrosis stage of non-tumorous liver in NBNC- HCC patients with Hepatic resection [striped square]:F0; $\square: F 1 ; \square: F 2 ; \square: F 3 ; \square: F 4$ 